\title{
Reactive Lesions of Oro-Maxillofacial Region
}

\author{
Raja Sekhar Gali
}

\subsection{Introduction}

The tissues of the oral and maxillofacial region are constantly exposed to a plethora of inflammatory stimuli that can be of bacterial, physical, chemical or immunologic in the origin. Chronic/long-standing, low-grade inflammatory stimuli can induce a heightened/escalated reparative response in the oral tissues resulting in the occurrence of nonneoplastic, hyperplastic lesions that are collectively referred to as reactive lesions [1].

Though the occurrence of exophytic reactive proliferation in the oral cavity is relatively common, certain malignant (metastatic/primary) and benign tumours, vascular lesions can closely mimic these lesions leading the clinician to misdiagnosis and sub-optimal treatment. A thorough understanding of the clinical presentation, differential diagnosis and the required diagnostic workup is the key to appropriate treatment.

\section{Additional features of the chapter: \\ - Pearls, Perils \& Pitfalls \\ - Case Scenarios \\ - Recent Advances}

\subsection{Classification}

These lesions can be peripheral/extra-osseous (Soft tissuesGingiva, oral mucosa etc.) or Central (intra-osseous) in occurrence.

\footnotetext{
R. S. Gali $(\bowtie)$
}

Department of Oral and Maxillofacial Surgery, Narayana Dental

College and Hospital, Nellore, Andhra Pradesh, India

Medicover Hospital, Nellore, Andhra Pradesh, India

\section{Peripheral reactive lesions:}

Eversole classified the common peripheral hyperplastic lesions of gingiva and other oral mucosal sites into the following categories $[2,3]$.

1. Pyogenic Granuloma

2. Peripheral Giant Cell Granuloma

3. Peripheral Ossifying Fibroma

4. Peripheral fibroma (Focal Fibrous hyperplasia/ Fibrous Epulis) [2].

\section{Central reactive lesions:}

The intra-osseous reactive lesions include the following categories $[4,5]$.

1. Central giant cell Granuloma

2. Aneurysmal Bone Cyst

3. Brown's Tumour of hyperparathyroidism

4. Chronic Sclerosing Osteomyelitis and Garre's osteomyelitis/Proliferative Periosteitis $[4,5]$.

\subsection{Pyogenic Granuloma}

\subsubsection{Clinical Features and Aetiopathogenesis}

Pyogenic Granuloma presents as a painless pedunculated or sessile, soft fleshy mass that bleeds easily with a normal or occasionally ulcerated overlying surface (Fig. 29.1). Gingiva is the most common intra-oral site, although it can affect lips, mucosa and tongue [3].

It is a localised proliferative mass occurring as a result of an exuberant reparative response to various stimuli such as chronic local irritation (calculus, overhanging restoration, a sharp margin of crown etc.), trauma, hormonal changes (Pregnancy), bone marrow transplants and reac- 


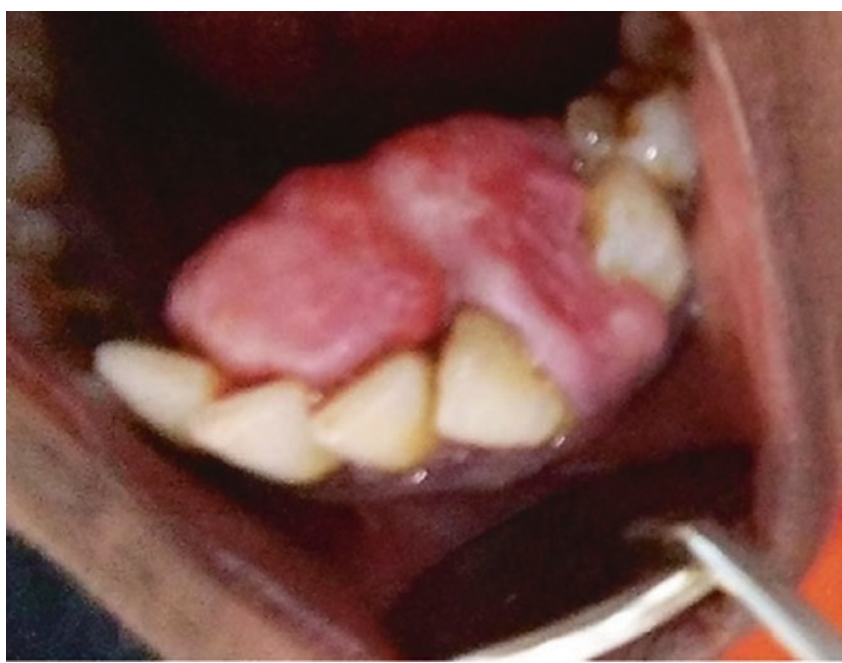

CAssociation of Oral and Maxillofacial Surgeons of India

Fig. 29.1 Exophytic gingival growth associated with chronic gingivitis with heavy bands of calculus. The lesion easily bleeds on palpation and can occasionally cause displacement of teeth

tion to grafts. Pulp polyp or hyperplastic pulpitis is also a type of pyogenic granuloma arising in response to lowgrade chronic infection [1]. The term pyogenic granuloma is a misnomer as it does not contain pus and it is not a true granuloma $[1,3]$.

It can occur across all the age groups and both genders, but with a distinct predominance in females in the 2 nd decade of life due to the increased levels of circulating hormones like oestrogen and progesterone [6]. Equal incidence has been reported in both the jaws with a slight predilection to anterior maxilla [1].

\subsubsection{Differential Diagnosis}

Other peripheral reactive lesions that resemble pyogenic granuloma are peripheral giant cell granuloma and peripheral ossifying fibroma [3]. Peripheral ossifying fibroma has a firm consistency and a lesser tendency to bleed. Specific proliferative malignant lesions can closely mimic pyogenic granuloma misguiding the clinician leading to sub-optimal treatment. These can be primary or metastatic squamous cell carcinomas, fibrosarcoma, Kaposi's sarcoma, oral metastatic deposits from distant primary foci like thyroid, lung kidneys etc [1].

\subsubsection{Diagnostic Workup}

Radiological Features Appreciable radiological changes are generally not seen in pyogenic granuloma, but occasion- ally cup-like resorption of underlying alveolar bone can be seen in a peri-apical view or an orthopantomogram. Ruling out the presence of large osteolytic lesions is required to exclude malignant lesions.

Histopathological Features Histologically pyogenic granulomas are classified into two distinct sub-types- the nonlobular capillary haemangioma type (non-LCH type) and Lobular capillary haemangioma (LCH) type [6]. The nonLCH type comprises of highly vascular proliferation that is similar to granulation tissue with foci of fibrous tissue whereas the LCH type shows proliferation of blood vessels that are aggregated in a lobular fashion without any signs of inflammation and or reparative response.

\subsubsection{Recent Concepts}

Epivatianos A et al. had demonstrated that these two subtypes have a different origin, clinical behaviour and immunohistochemical properties [7]. Their study had shown that non-LCH pyogenic granuloma presented clinically as a pedunculated mass and was mostly associated with identifiable aetiologic factors and showed a histological picture of a reparative response. However, LCH pyogenic granuloma presented as a non-ulcerated sessile mass without any identifiable aetiology and histological evidence of lobulated vessel pattern and the absence of inflammatory reaction. This has led to the concept that $\mathrm{LCH}$ pyogenic granuloma represents a benign tumour of the vascular origin that develops due to unknown factors just like other benign tumours.

\subsubsection{Treatment}

Surgical excision of the lesion followed by removal of local irritants such as calculus, foreign body etc. is the standard treatment. Excision up to the periosteum is required in gingival lesions followed by either primary closure by mobilisation of adjacent mucoperiosteum, or surgical defect is left to heal by secondary intention by the placement of Coe pack. Brisk bleeding is often encountered during excision that would require electrocoagulation. Other treatment options include electrocautery, cryosurgery, sclerotherapy and laserassisted excision.

\subsubsection{Prognosis}

Complete excision of the lesion till the periosteum and removal of local aetiological factors will eliminate the chances of recurrence. 


\subsection{Peripheral Giant Cell Granuloma}

Peripheral giant cell granuloma is the most commonly encountered giant cell containing lesion of the oral cavity that closely resembles pyogenic granuloma.

\subsubsection{Clinical Features and Aetiopathogenesis}

It is thought to originate mainly from the connective tissue of the gingiva or the periosteum of the alveolar ridge. It has been reported in all age groups but with a peak incidence in the $3 \mathrm{rd}$ and 4 th decades of life with a female predominance [8]. The lesion presents as a soft proliferative mass occurring at gingiva or even at edentulous alveolar ridge. It has a tendency to bleed and may or may not be pedunculated [9].

The term 'reparative' was dropped from its nomenclature as the reparative nature could not be demonstrated histopathologically. The aetiology of this lesion remains unclear, but irritating local factors such as poor restorations, prostheses calculus etc. are thought to contribute to its origin (Fig. 29.2).

\subsubsection{Differential Diagnosis}

Clinically pyogenic granuloma and peripheral giant cell granuloma are indistinguishable. Erosion of cortical bone is more commonly seen with peripheral giant cell granuloma than with pyogenic granuloma.

Histopathologically, the lesion is very similar to central giant cell granuloma, its intra-osseous counterpart [9].

\subsubsection{Radiologic Features}

Since the lesion is peripheral in the origin and location, radiologic changes are rarely seen. Longstanding, larger lesions can exhibit superficial cortical erosion and may also show widening of adjacent periodontal ligament space.

\subsubsection{Histopathological Features}

Hyperplasia of fibroblasts with abundant multi-nucleated giant cells, with scattered areas of chronic inflammatory cells and neutrophils are seen [9]. Controversy exists about the origin of the giant cells; some believe that they are of the osteoclast origin while others state that they are formed by the fusion of mono-nuclear cells. Irrespective of their origin, they are non-functional in these lesions as they are not involved either in phagocytosis or bone resorption [9].

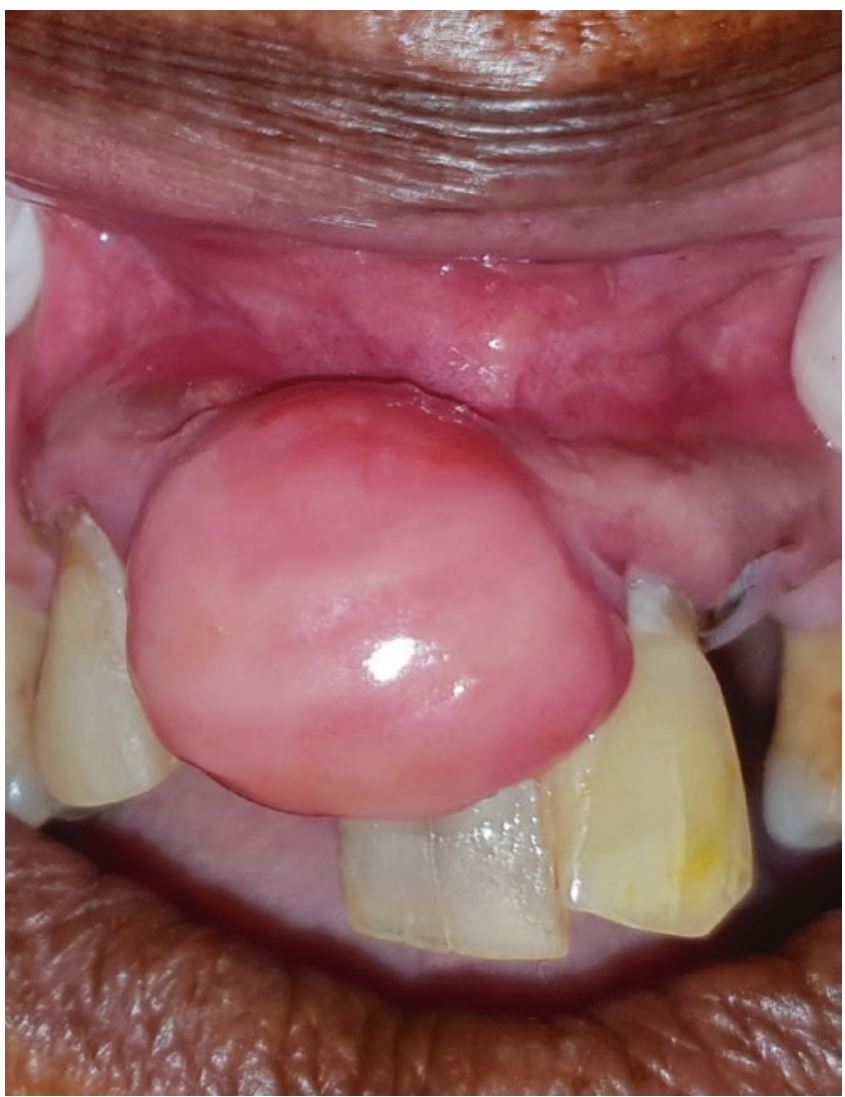

CAssociation of Oral and Maxillofacial Surgeons of India

Fig. 29.2 Peripheral giant cell granuloma, presenting as an exophytic gingival growth firm to hard in consistency causing displacement of adjacent teeth

\subsubsection{Treatment}

Complete surgical excision including the periosteum or periodontal ligament as applicable followed by the removal of local factors is the optimum treatment for peripheral giant cell granuloma. Recurrences are rare, but have been reported and are believed to be due to non-inclusion of periosteum or periodontal ligament during excision.

\subsubsection{Prognosis and Complications}

Recurrence of PGCG is infrequent, ranges as little as 5-11\% have been reported. Extensive clearing of the base of the lesion, eradication of the source of irritation prevents recurrence [9].

\subsubsection{Recent Advances}

Increasing incidence of peripheral giant cell granulomas occurring in association with dental implants has been reported $[10,11]$. Local irritant factors, inflammation of 
peri-implant tissues are the causative factors. Clinically, these proliferations are similar to the classic peripheral giant cell granulomas and usually develop within months to years following placement of dental implants. If early detection and complete surgical excision are not performed, such lesions can lead to peri-implantitis, ultimately leading to failure of the implant necessitating its removal.

\subsection{Peripheral Ossifying Fibroma}

The peripheral ossifying fibroma is a reactive gingival proliferation presenting as nodular mass firm in consistency with or without ulceration. It contains fibroblastic cellular tissue with varying amounts of mineralised content that can be either bone, cementum or dystrophic calcification.

The term 'peripheral ossifying fibroma' literally means a benign tumour of fibrous connective tissue origin. However, this lesion is a non-neoplastic reactive proliferative lesion, so this term is a misnomer for this pathology [12].

\subsubsection{Clinical Features and Aetiopathogenesis}

The lesion presents as a slow-growing solitary gingival growth commonly seen during the second decade of life, predominantly in women in the anterior region of either of the jaws. The lesion is believed to originate from periodontal ligament in response to local irritation or inflammation, commonly seen at the inter-dental region as a pedunculated or sessile mass (Fig. 29.3). The exact pathogenesis of peripheral ossifying fibroma is controversial. Some authors believe that it initially develops as a pyogenic granuloma that subsequently undergoes fibrous maturation and calcification. It is thought that these two lesions represent progressive stages of the same reactive pathology [13].

\subsubsection{Differential Diagnosis}

Histopathology is the only method by which one can distinguish a peripheral ossifying fibroma from a pyogenic granuloma or peripheral giant cell granuloma [14].

\subsubsection{Radiologic Features}

Radiographs may occasionally demonstrate specks of calcifications in these lesions (Fig. 29.4a-c).

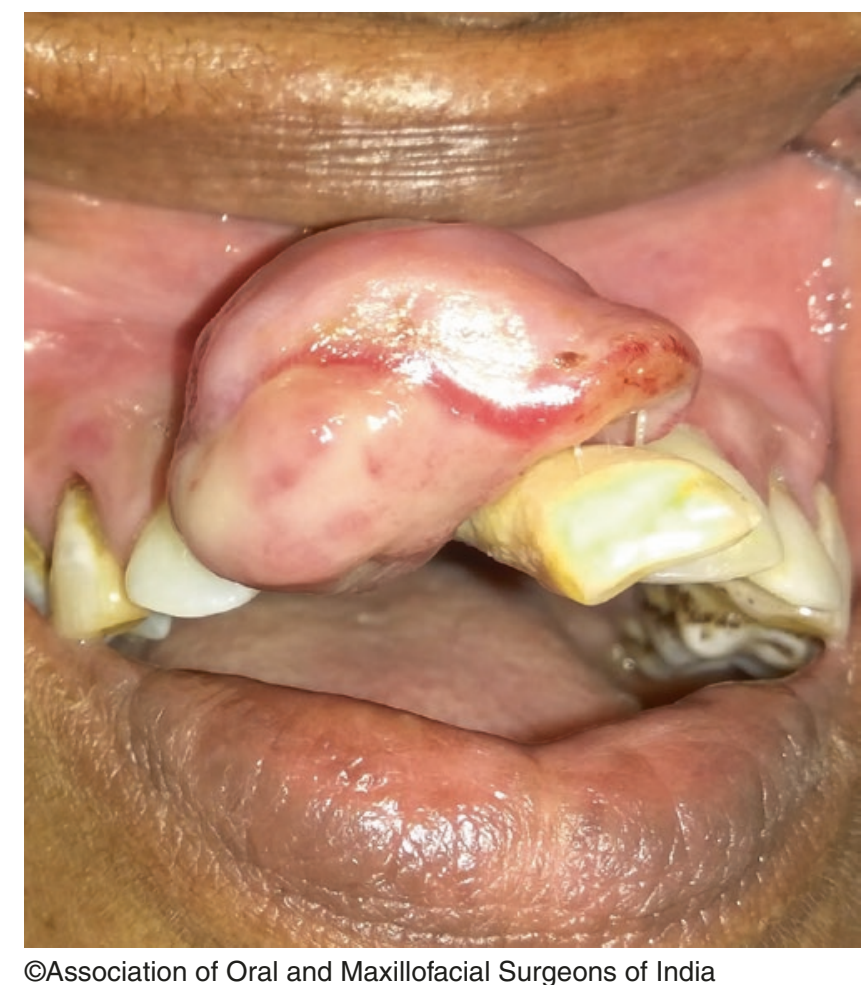

Fig. 29.3 Exophytic sessile mass over gingiva at anterior maxilla associated with heavy bands of calculus and is seen to be causing displacement of teeth

\subsubsection{Histopathologic Features (Fig. 29.4c)}

The peripheral ossifying fibroma consists of rich fibro-cellular tissue with focal areas of mineralised deposits containing bone, cementum or dystrophic calcification. A chronic inflammatory cell infiltrate is observed at the periphery of the lesion.

\subsubsection{Treatment}

Complete surgical excision of the lesion with circumferential margins of $3 \mathrm{~mm}$ of healthy gingival tissue and deep margins down to the bone, including the adjacent periosteum and periodontal ligament has to be performed to reduce the chances of recurrence [1]. Extraction of the involved teeth is to be considered in the cases of recurrent or multi-focal lesions of peripheral ossifying fibromas.

\subsubsection{Prognosis and Complications}

The reported reccurrence rate for peripheral ossifying fibroma is surprisingly high for a reactive proliferation. The recurrence rate has varied from 4 to $75 \%[15,16]$. 


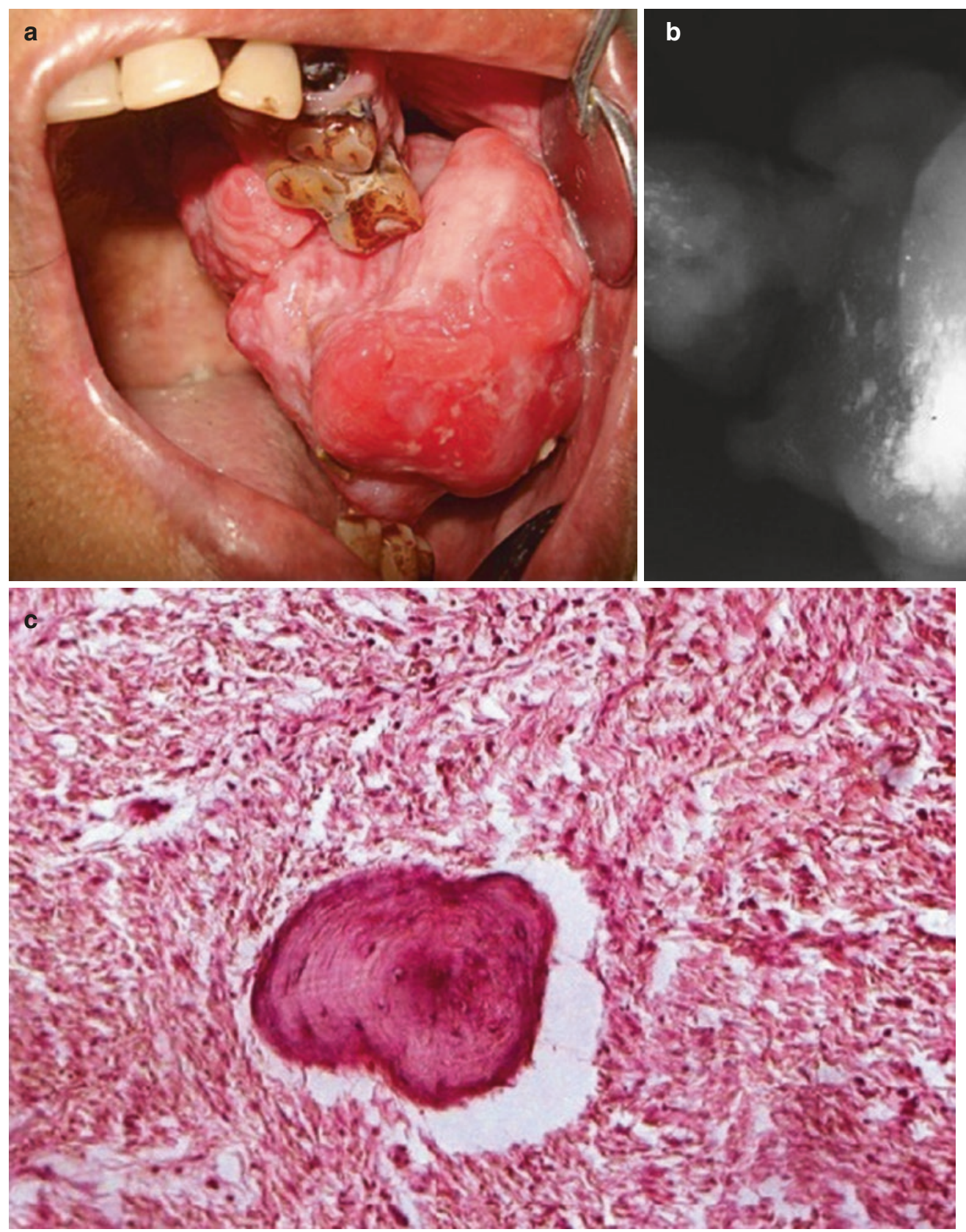

CAssociation of Oral and Maxillofacial Surgeons of India

Fig. 29.4 (a) Large peripheral ossifying fibroma of $6 \times 7 \mathrm{~cm}$ in size, in a 64-year-old female, 5 years duration presenting as firm mass. (b) Radio-opacities on the radiograph observed in the excised lesion. (c) Histopathological view showing the woven bone (H\&E stain, original magnification 400×). (Adapted from A rare massive exophytic gingival growth: Suvy Manuel et al.; Clinics and Practice. 2012 Mar 30; 2(2): e38. Open Access DOI https://doi.org/10.4081/cp.2012.e38 , copy right S. Manuel et al, CC BY-NC 3.0, Licensee PAGEPress Italy) 
Pearls, Perils and Pitfalls: Peripheral Reactive Lesions:

1. The most common proliferative masses of Gingiva are a Triad of reactive lesions-The 3 P'sPyogenic Granuloma, Peripheral giant Cell granuloma \& Peripheral Ossifying fibroma.

2. Pyogenic granulomas and peripheral giant cell granulomas are more vascular, which is reflected by their tendency to bleed and their red or reddish-blue colour.

3. Unlike pyogenic granuloma, peripheral giant cell granuloma can occur on edentulous alveolar ridges arising from the periosteum

4. POF is firm to hard in consistency due to the mineralised content like cementum/bone/dystrophic calcification

5. Complete Surgical excision of these lesions with circumferential margins of $3 \mathrm{~mm}$ of healthy gingival tissue and deep margins down to the bone, including the adjacent periosteum \& periodontal ligament has to be performed to reduce the chances of recurrence. This should be followed by removal of putative local factors like calculus, plaque faulty restoration/prosthesis etc.

6. Extraction of the involved but firm teeth should be considered in the cases of recurrent/multi focal lesions.
The aggressive clinical behaviour of specific lesions demonstrated by osteolysis, cortical expansion, perforation and extension into soft tissue has led to the view that it is a benign tumour similar to the giant cell tumour of long bones [18]. The exact relationship between these two pathologies remains to be understood. The concept that these lesions represent two points in the spectrum of a single-disease process has been putforth $[19,20]$. Considering the variability in clinical presentation, individual authors have classified it as a rapidly growing \& destructive-aggressive form and a slow-growing, incidentally picked up lesion-the nonaggressive form $[19,20]$.

Since the true neoplastic nature is yet to be established and the histologic features do not represent a true granulomatous lesion, the term central giant cell 'lesion' had been proposed by Whitaker \& Waldron [20].

\subsubsection{Clinical Features and Aetiopathogenesis}

The lesion can occur in any age group but, children and young adults are more commonly affected and with a female predominance [19]. The anterior mandibular region is the most frequent site of occurrence almost twice as compared to the maxilla. The most common presentation is that of an asymptomatic, slow-growing, bony hard and expansile swelling of the involved jaw [19, 20]. This can be accompanied by progressive displacement/loosening of teeth, paresthesia, nasal obstruction and secondary infection leading to pain (Fig. 29.5a, b).

\subsubsection{Differential Diagnosis}

Histopathologically, central giant cell granulomas and brown tumour of hyperparathyroidism are indistinguishable from each other, and this can be very misguiding to the clinician and the pathologist. Hence, biochemical tests like serum calcium, phosphate and parathormone levels are to be evaluated to rule out brown's tumour. In all cases of multi-focal and or recurrent central giant cell granulomas of jaws, the possibility of primary or secondary hyperparathyroidism is to be ruled out, so that unnecessary surgical procedures and their added morbidity can be avoided.

\subsubsection{Radiological Features}

The central giant cell granuloma presents as multi-locular radiolucency with scalloped margins that are usually noncorticated [19]. The cortical expansion, thinning followed by perforation, root resorption and displacement of adjacent teeth can be seen in aggressive variants [20] (Fig. 29.5c, d). 

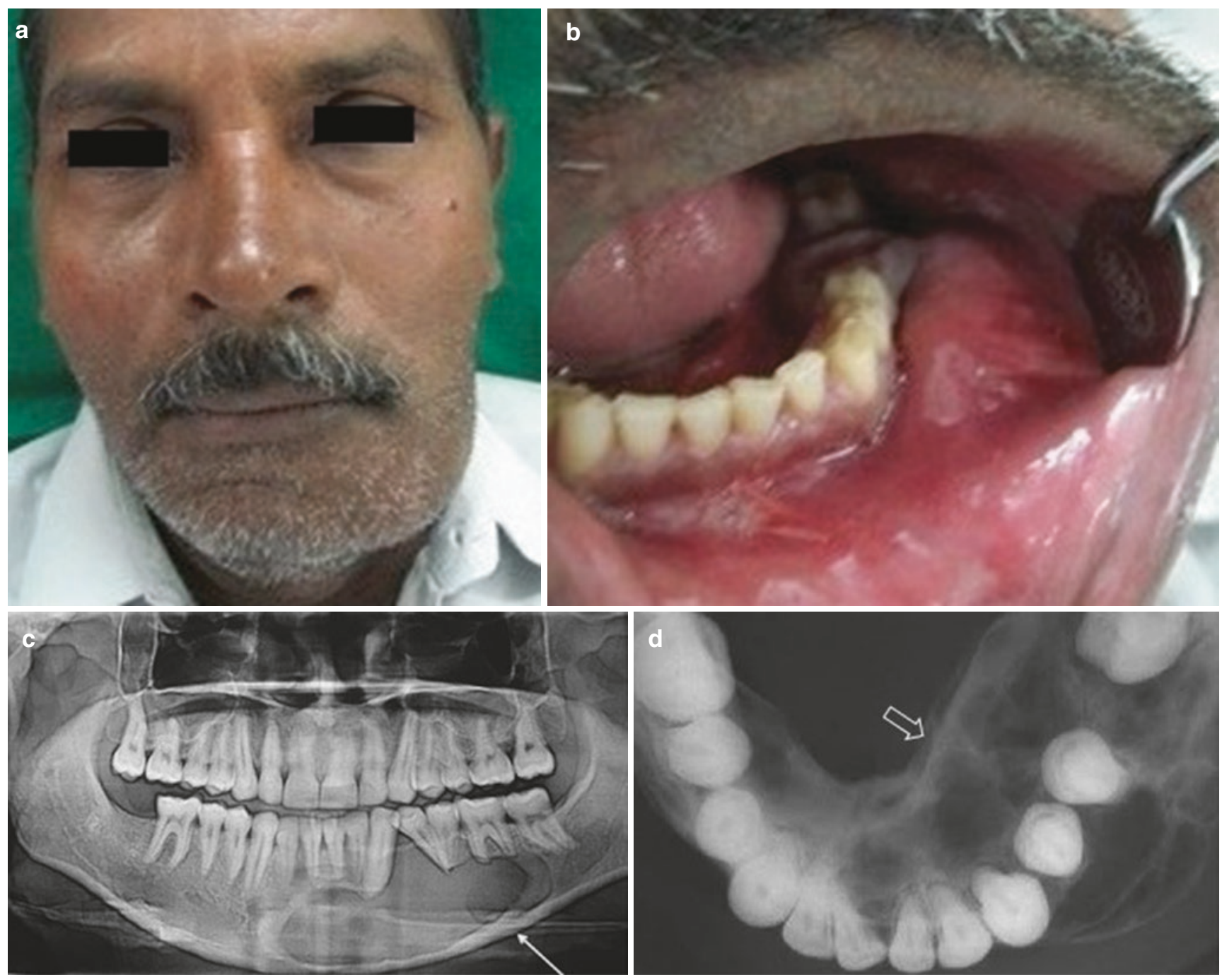

CAssociation of Oral and Maxillofacial Surgeons of India

Fig. 29.5 (a, b) Central Giant cell granuloma presenting as hard bony mass over the left body of the mandible with bi-cortical expansion. (c) Central Giant cell granuloma presenting as a unilocular osteolytic

\subsubsection{Histopathologic Features}

The lesion consists of a proliferation of spindle-shaped fibroblasts in fibrous and fibromyxoid/vascular stroma [20]. Clusters of multi-nucleated giant cells are scattered throughout connective tissue stroma especially around vascular channels. These cells are believed to originate from osteoclasts or by the fusion of mono-nuclear cells [9]. Focal deposits of metaplastic bone are seen. The histological picture is precisely identical to brown's tumour of hyperparathyroidism [1].

\subsubsection{Treatment}

The most commonly practised and favoured treatment for central giant cell granuloma has been aggressive surgical lesion with sclerotic border and thinning of lower border. (d) Occlusal radiograph demonstrating bi-cortical expansile lytic lesion with incomplete septae

curettage. Recurrence rates ranging from 20 to $50 \%$ have been reported following curettage [21]. Adjuvant treatments to curettage-like peripheral ostectomy or marginal resection have been advocated to reduce the chances of recurrence [22]. Aggressive surgical treatment options such as en bloc resection/segmental mandibulectomy/ maxillectomy are presently reserved for locally advanced lesions \& recurrent lesions [22, 23]. Significant vascularity and intra-operative bleeding are frequently associated with these lesions. Aggressive surgical resection with $0.5 \mathrm{~cm}$ margins of healthy tissue is advocated to reduce the chances of recurrence [24]. However, the morbidity associated with surgical resection such as functional, cosmetic impairment, loss of teeth, teeth buds and neurosensory disturbances questions the need for such aggressive treatments in a benign reactive lesion-like central giant cell granuloma [24]. 

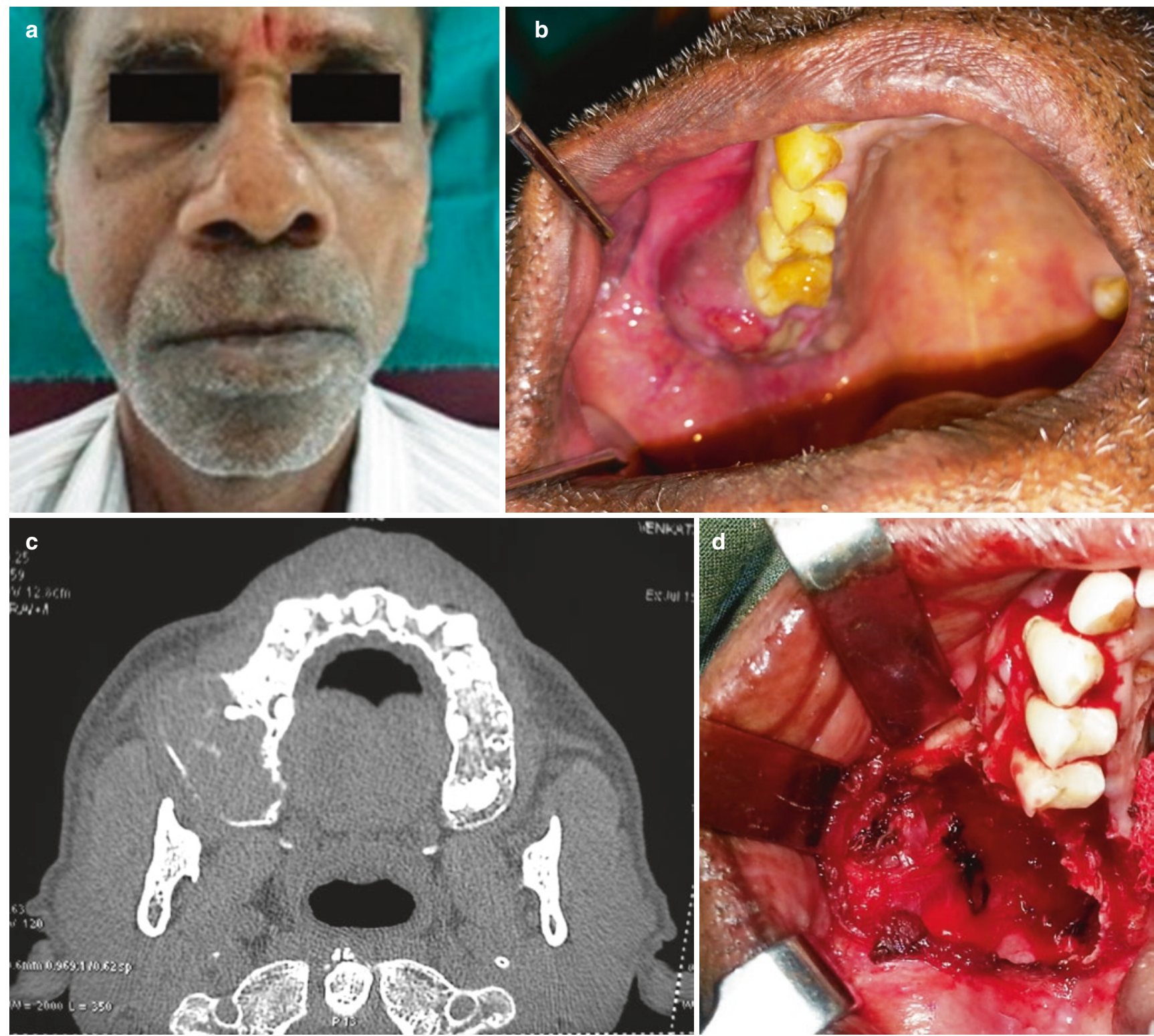

CAssociation of Oral and Maxillofacial Surgeons of India

Fig. 29.6 (a) Swelling right side face. (b) Intra oral view of lesion right posterior maxilla. (c) CT axial cut showing changes in posterior right maxilla. (d) Intra operative view

Medical Methods of Treatment Based on the premise that osteoclastic activity steers the progression of the disease process, medical treatments aimed at antagonising the activity and proliferation of osteoclasts have been used with varying rates of success [25-27].

Intra-lesional Steroids Steroids have demonstrated the ability to inhibit bone resorption and induce apoptosis of osteoclasts. Weekly intra-lesional injections of triamcinolone acetonide for six weeks have shown highly variable results from partial shrinkage, in complete remission, to complete resolution leading to hypercalcification of the area $[28,29]$. The protocol described involves the use of a $50 \%$ mixture of local anaesthetic and $10 \mathrm{mg} / \mathrm{ml}$ triamcinolone acetonide. Two $\mathrm{ml}$ of this solution has to be used for every $1 \mathrm{cc}$ of the lesion visible on orthopantomogram $[29,30]$.

Systemic Calcitonin Therapy Based on the observations that calcitonin receptors were found in the multi-nucleated giant cells of central giant cell granuloma and the role of calcitonin in inhibiting osteoclast function, systemic administration of calcitonin has been practised to treat central giant cell granuloma [31]. Calcitonin is administered through a sub-cutaneous or intra-nasal route. Subcutaneous administration involves 100 units per day for a period of 18-21 months during which sequential radiographs are obtained to monitor the remission of the lesion. 
Unlike sub-cutaneous administration, the intra-nasal route has better patient compliance but unpredictable absorption rates [31].

Results with calcitonin therapy have been highly variable. In some instances, they have been used to shrink the lesions first, later to be followed by curettage.

Interferon Therapy Owing to the prominent vascular component in central giant cell granuloma, the anti-angiogenic properties of interferon alpha have been used as adjuvant therapy following enucleation/curettage of the lesion. Isolated interferon therapy without prior surgical procedure led to partial resolution of lesions and is of limited value. Kaban et al. had proposed a protocol that involved treatment of aggressive central giant cell granulomas of the mandible by a staged treatment of conservative surgical enucleation (sparing the inferior alveolar nerve and teeth). This is followed by administration of interferon Alpha 2 or beta $\left(3,000,000\right.$ units $\left./ \mathrm{m}^{2}\right) 48-72 \mathrm{~h}$ after surgery, given once daily by a sub-cutaneous route for a period of 6 months or till a period when CT scan showed complete regeneration of bony defect [32]. Their study was based on the hypothesis that these lesions are vascular proliferations, thus explaining the high success rate in response to anti-angiogenic therapy in their experience [32].

Bisphosphonates Bisphosphonates that have an antagonising effect on the osteoclastic activity. Owing to the virtue of this property, bisphosphonates have been used in conjunction with intra-lesional steroids in central giant cell granuloma with promising results [33].

Denosumab Denosumab is a human monoclonal antibody that binds to the RANK ligand located on the surface of osteoclasts and deactivates them. The stromal cells of giant cell tumour have been shown to secrete RANKL and upregulate osteoclast activity and development. Recent Phase-II clinical trials on the use of denosumab in giant cell tumours of long bones showed that it reduces the need for morbid surgeries [34, 35]. A single report on the use of denosumab in central giant cell granulomas of jaws in two patients was recently published and had claimed very promising results involving complete remission of jaw lesions. However, studies involving a larger sample with longer follow-up periods are awaited to know the exact risk-benefit status of this novel treatment option [36].

\subsection{Aneurysmal Bone Cyst (Fig. 29.7a-f)}

The aneurysmal bone cyst is a controversial lesion believed to be a non-neoplastic and exaggerated reactive response of vascular tissues of bone. It is a pseudocyst that presents as expansile, osteolytic lesion often characterised by ballooning distension of the cortex, that consists of blood-filled cavernous spaces showing occasional osteoid material and multi-nucleated giant cells [37].

\subsubsection{Clinical Features}

The aneurysmal bone cyst generally occurs in patients less than 30 years of age with a reported higher incidence during the second decade of life with almost equal gender predilection. Aneurysmal bone cysts of the jaws are relatively rare comprising of only $1-3 \%$ of all aneurysmal bone cysts occurring in the skeleton. The posterior region of the mandible is the preferred site compared to maxilla $(6: 1)$.

Most of these lesions present as expansile swellings with or without perforation of the involved jaw bone, causing facial asymmetry or displacement of adjacent teeth and malocclusion. Pain, paresthesia and rapid progression of swelling can be seen occasionally. Dark venous blood is generally obtained on aspiration (Fig. 29.7a-c).

Pulsatile nature, thrill and bruit are generally not seen in these lesions despite the prominent vascular component associated with aneurysmal bone cysts. The presence of these signs in an expansile, osteolytic jaw lesion would rather indicate a high flow vascular malformation. The clinician should recognise this and not mistake it for an aneurysmal bone cyst, because doing an aspiration or incisional biopsy of such high flow vascular malformations can lead to disastrous bleeding and potentially fatal outcomes.

\subsubsection{Aetiopathogenesis}

The exact mechanism regarding the origin of aneurysmal bone cysts remains controversial. The reported hypotheses include post-traumatic, reactive malformation, genetic predisposition causing chromosomal translocation and minor vascular injury in a pre-existing bone lesion leading to pooling of blood in the stromal spaces creating bone destruction [38].

This lesion can occur de novo as a primary aneurysmal bone cyst or as a secondary phenomenon arising in preexisting lesions like central giant cell granuloma, ossifying fibroma, fibrous dysplasia, osteosarcoma, fibrosarcoma and other lesions [38]. It has been postulated that injury to the capillary network in these lesions leads to extravasation of blood. The associated capillary blood pressure readily destroys unsupported stroma resulting in a blow out reaction thereby producing the expansile and destructive aneurysmal bone cyst. 

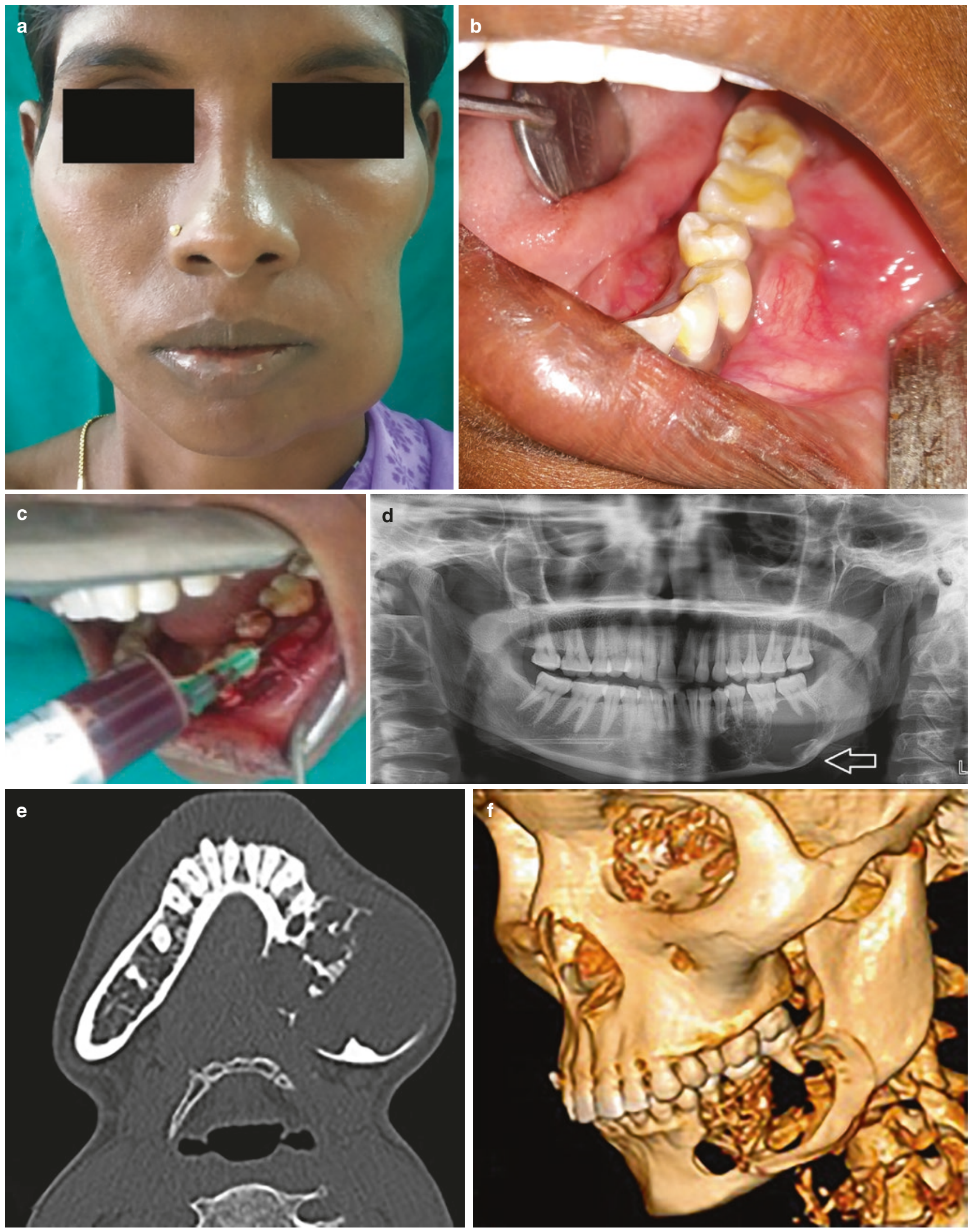

CAssociation of Oral and Maxillofacial Surgeons of India

Fig. 29.7 (a) Swelling left side body mandible. (b) Intra oral view of swelling left lower posterior buccal cortex. (c) Aspirate showing blood tinged fluid. (d) OPG showing lesion left body mandible. (e) Axial CT scan showing the expansile lesion. (f) 3D CT showing the destruction of bone 


\subsubsection{Radiologic Features}

Owing to its osteolytic nature, aneurysmal bone cyst presents as unilocular or multi-locular radiolucency often associated with cortical thinning and perforation with resorption of roots seen occasionally. A classic sign commonly seen is the 'ballooning' distension of the thinned out cortex occurring as a result of a thin outline of reactive sub-periosteal bone formation. CT and MRI scans of such lesions often demonstrate blood and free fluid in these cavities (Fig. 29.7d, e, f).

These typical features can sometimes be masked in those aneurysmal bone cysts occurring as secondary phenomena in pre-existing bone lesions. Such aneurysmal bone cysts present as mixed radiolucent-radiopaque lesions.

\subsubsection{Histopathological Features}

Histopathologically, it consists of variable amounts of fibrous connective tissue stroma with blood-filled sinusoidal spaces, multi-nucleated giant cells and reactive woven bone/osteoid. Correlating the histopathological and clinical features, three variants of aneurysmal bone cysts were proposed namely, the solid type, vascular type and mixed type [39]. The solid type is associated with a dense stroma, few sinusoidal spaces and blood vessels that correlates with the absence of cortical perforation clinically and minimal bleeding observed intra-operatively. The vascular variant falls into the other end of the spectrum that shows loose fibrous tissue stroma with large blood-filled cavernous spaces causing extensive osteolysis and cortical perforation that is frequently associated with brisk intra-operative bleed. The mixed variant shows characteristics of both these sub-types.

\subsubsection{Treatment}

Complete Surgical curettage of the lesion is the most commonly followed treatment modality. Adjuvant therapies like cryosurgery and electrocauterisation have been used to reduce the chances of recurrence. Due to its vascular nature, brisk bleeding is often encountered during surgery that generally ceases after complete removal of the lesion. Preoperative embolisation had been advocated in extensive, vascular variants of aneurysmal bone cysts to reduce intraoperative blood loss.

Resection is generally reserved for aggressive aneurysmal bone cysts that have extensively resorbed the lower border of the mandible or have caused a pathological fracture and lesions that have recurred following conservative therapy. The decision to perform segmental resection is also influenced by the nature and extent of primary jaw pathology in which the aneurysmal bone cyst has arisen. Segmental resection has been recommended as the treatment option for sec- ondary aneurysmal bone cysts occurring in ossifying fibromas, giant cell granulomas and osteoblastomas [40].

\subsubsection{Prognosis and Complications}

Aneurysmal bone cysts have relatively higher recurrence rates compared to central giant cell granuloma [38]. This has been attributed to incomplete removal of the lesion during curettage/marginal mandibulectomy. The multi-locular nature of the lesion can sometimes limit the access leading to residual pathological tissue that causes increased intraoperative bleeding and also gives rise to recurrences usually in the first year following surgery.

\subsection{Brown Tumour of Hyperparathyroidism (Fig. 29.8)}

Brown tumour is a misnomer as it is not a true neoplasm, but a localised area of osteolytic defect caused by increased osteoclastic activity and demineralisation of bone that is induced by excessive secretion of parathyroid hormone. It is considered to be an intra-osseous reactive lesion [4].

\subsubsection{Etiopathogenesis}

Hypersecretion of parathyroid hormone can occur due to Primary (benign/malignant adenoma of parathyroid gland), secondary (pre-existing kidney disease leads to compensatory overactivity of parathyroid) or tertiary causes (parathyroid tumours arising due to prolonged hyperactivity induced by secondary causes). Irrespective of the origin, the resultant effect is hypercalcaemia (caused by mobilisation of calcium from bone to bloodstream) and altered phosphate levels that give rise to the entire spectrum of signs and symptoms of this disease. These include disturbances in ion metabolism, demineralisation of bone, kidney stones, gastrointestinal disorders and muscle weakness. Hence, hyperparathyroidism is also referred to as the 'disease of bones, stones, abdominal groans and psychic moans' [41].

\subsubsection{Clinical Features}

Brown tumour is a localised, lytic bone defect characterised by a mass of reactive fibrous tissue that presents as swelling with cortical expansion, perforation occasionally associated with pain and pathological fracture. The lesion occurs in adults above 50 years of age and has a female to the male pre-dilection of 5:2 and can occur in any bone but more common sites are long bones, pelvis, ribs, maxilla and mandible [42]. Co-existing multiple synchronous lesions in the jaws and at other skeletal sites can also occur 
Fig. 29.8 (a) Lesion right posterior maxilla. (b) Lesion anterior mandible. (c) OPG showing the changes (red circles)
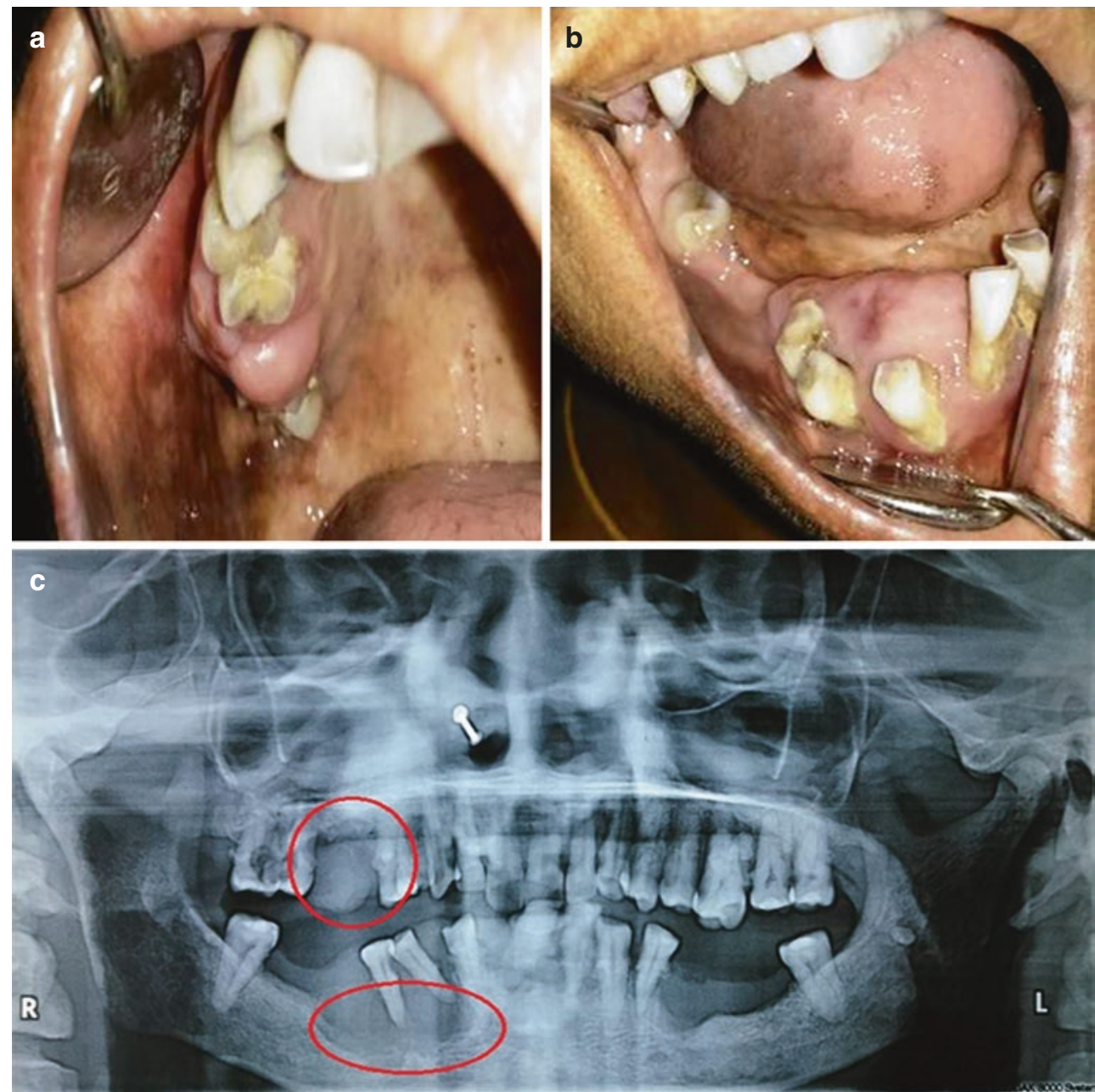

CAssociation of Oral and Maxillofacial Surgeons of India
[43] (Fig. 29.8a-c). Bone manifestations usually occur in the late stages of hyperparathyroidism, and brown tumours are picked up during the diagnostic work-up for the same. Rarely, brown tumour of the jaw may be the first manifestation of primary hyper parathyroidism [44, 45]. They can cause significant bone destruction leading to facial asymmetry, displacement of teeth, malocclusion pathological fracture etc.

\subsubsection{Diagnostic Workup}

Since it is not possible to histopathologically distinguish brown tumour from central giant cell granuloma, biochemical tests are mandatory to confirm its diagnosis. Elevated serum calcium $(>10.4 \mathrm{mg} / \mathrm{dl})$, decreased serum phosphate level, elevated parathormone levels, ultrasonography to identify parathyroid adenomas (primary hyperparathyroidism), and renal function tests to rule out renal disease (secondary hyperparathyroidism) constitute the sequence of diagnostic work up to diagnose hyperparathyroidism [45].

\subsubsection{Differential Diagnosis}

- Giant cell granuloma

- Giant cell tumour

- Aneurysmal bone cyst

\subsubsection{Radiographic Features}

It shows a well-defined mono-locular or multi-locular welldemarcated lesion that is purely lytic. There are significant root resorption and loss of lamina dura.

\subsubsection{Histopathology}

Brown tumour very closely resembles central giant cell granuloma, and these two lesions are indistinguishable on histopathology. Features such as the proliferation of multi-nucleated giant cells, osteoclasts present in a rich fibro-cellular and vascular connective tissue stroma with 
scattered areas of haemorrhagic foci and haemosiderin pigmentation are seen. The presence of haemosiderin renders this lesion its characteristic brown/brownish yellow colour and hence the name brown tumour.

\subsubsection{Treatment}

Spontaneous resolution/remission of brown tumour generally occurs after treatment of the underlying cause of hyperparathyroidism. Primary hyperparathyroidism is treated by excision of parathyroid adenoma/gland. Treatment of renal disease, vitamin D deficiency is done in the cases of secondary hyperparathyroidism. Refractory lesions that fail to resolve or incomplete remissions after treatment of underlying cause would require surgical curettage $[44,45]$.

\subsubsection{Prognosis}

Brown tumours are generally slow-growing lesions that can be locally destructive. Invasion into surrounding structures may cause a variety of symptoms. Small lesions usually resolve spontaneously once the underlying condition (hyperparathyroidism) is treated whereas large lesions take about 6-12 months to subside.

Pearls, perils and pitfalls:

1. Central Giant cell granuloma (CGCG) and brown tumour are indistinguishable histologically. An incisional biopsy report of CGCG should prompt the clinician to ask for biochemical investigations to rule out the brown tumour, that is characterised by increased serum levels of calcium, phosphate, alkaline phosphatase and parathyroid hormone.

2. It is crucial to diagnose brown tumours that are invariably reported as CGCG on incisional biopsy because treatment of hyperparathyroidism will generally lead to spontaneous resolution of brown jaw tumours, thereby avoiding unnecessary surgical procedures of jaw lesions.

3. Aneurysmal bone cysts have varied clinical, radiological and histological presentations.

4. Secondary aneurysmal bone cysts that arise in preexisting pathologies have a higher tendency to recur if treated by conservative surgical methods like curettage.

\subsection{Chronic Sclerosing Osteomyelitis and Garre's Osteomyelitis}

Chronic sclerosing type of osteomyelitis occurs as a result of low-grade and long-standing infection of the marrow spaces that is characterised by areas of reactive bone sclerosis extending over a larger area (diffuse sclerosing) or localised to one or two teeth (focal sclerosing) [4].

Garre's osteomyelitis also known as proliferative periostitis is a sub-type of osteomyelitis that occurs in children. It is characterised by reactive sub-periosteal bone formation in response to chronic low-grade infection. Predominantly occurring in the posterior region of the mandible, it is best seen on an occlusal radiograph or an axial section CT scan as onion skin-layered appearance of the expanded bony cortex.

Rarely, malignancies like osteosarcoma (juxtacortical and parosteal), chondrosarcoma, fibrosarcoma and others can closely mimic Garre's osteomyelitis leading to a diagnostic dilemma and sub-optimal treatment.

These lesions are discussed in detail in other chapters of the book.

\subsection{Conclusion}

A variety of reactive soft tissue and bony pathologies occur in the oro-facial region that arises in response to chronic lowgrade inflammation, trauma, metabolic and hormonal influences. These lesions very closely mimic benign and malignant neoplasms in their clinical, radiological and histological presentations. Knowledge about their etiopathogenesis, clinical behaviour patterns is of paramount importance for providing appropriate treatment.

\section{Case Scenario 1 (Fig. 29.5)}

A 53-year-old male patient reported with a chief complaint of bony hard swelling over the left side lower jaw of 8 month duration.

\section{Clinical Examination}

Bony hard swelling over the left body of the mandible was noted with the expansion of buccal and lingual cortical plates (Fig. 29.5a, b).

\section{Pre-operative Diagnostic Work-up}

Imaging: Orthopantomogram revealed unilocular radiolucency with a sclerotic border and X-ray occlusal view showed a bicortical expansile, osteolytic lesion at the left body of mandible, with areas of osteosclerosis (Fig. 29.5c, d). 
Incisional Biopsy: Incisional biopsy done under local anaesthesia proved it to be central giant cell granuloma. Biochemical tests for assessment of serum parathormone, calcium, phosphate levels were performed, and the possibility of brown tumour of hyperparathyroidism was ruled out.

Diagnosis: Central Giant cell granuloma of mandibular body.

Surgical Plan: Considering bi cortical perforation and thinning of lower border caused by the lesion, segmental mandibulectomy through submandibular approach + mandibular reconstruction plate fixation was performed.

Postoperative Period: Post-operative period was uneventful. The patient was last seen three years after surgery and was found to be recurrence-free.

\section{Case Scenario 2 (Fig. 29.6a-d)}

A 60-Year-old male with a complaint of an asymptomatic slow-growing swelling in the upper jaw of 10 months duration.

\section{Clinical Examination}

A single firm to hard swelling measuring approximately $4 \times 3 \mathrm{~cm}$ located over the right maxillary posterior alveolus causing buccolingual expansion, vestibular obliteration was found. It was extending from the mesial aspect of right maxillary 1 st molar to the maxillary tuberosity area. The swelling was non-tender, firm to hard in consistency, with normal overlying mucosa. There was associated odontogenic focus of sepsis, but there was grade-II mobility of right maxillary 1st molar.

\section{Pre-operative Diagnostic Work-up}

Imaging: A pre-operative CT scan revealed a unilocular radiolucency with scattered areas of radio-opacity located at right maxillary posterior alveolus that was causing buccal cortical expansion, thinning and even perforation with denuding of the roots of the 1st molar. Superiorly the lesion was found to be extending till the body of the zygoma and just abutting the floor of the maxillary sinus.

Histopathological and Biochemical Evaluation: An incisional biopsy of the lesion was performed under local anaesthesia, that was reported as a central giant cell granuloma of the maxilla. A thorough biochemical evaluation was done to rule out brown tumour of hyperparathyroidism as both the lesions are indistinguishable histopathologically. Serum calcium, parathormone, and phosphate were within the normal limits.

Diagnosis: Central Giant cell granuloma-Right maxillary posterior alveolus.

Surgical Plan: Extended alveolectomy of the right posterior maxillary alveolus + nasal antrostomy + two-layered closure of the resultant defect with oroantral communication by mobilisation of the buccal fat pad and adjacent Buccopalatal mucoperiosteum. Following excision of the lesion, since maxillary sinus was breached, the resultant defect was packed with ribbon gauzed soaked in whitehead's varnish, and a trans nasal inferior meatal antrostomy was done, through which the tip of the pack was brought outside and knotted for removal on the 12th day. A two-layered watertight closure of the wound was achieved.

Post-operative Period: The wound healed uneventfully. The patient was last seen 4 years post-operatively and was free of disease.

\section{Case Scenario 3 (Fig. 29.7)}

A 38-year-old female with a complaint of swelling at the left side lower jaw of 1-year duration.

\section{Clinical Examination}

Figure 29.7a A single firm to hard swelling was located over the left body of mandible, non-tender with normal overlying skin \& mucosa. On palpation, buccal and lingual cortical plate expansion (Fig. 29.7b) with eggshell crackling signpositive suggestive of expanded and thinned out cortical plates causing microfractures on digital pressure.

\section{Pre-operative Diagnostic Work-up}

Imaging: Orthopantomogram revealed a radiolucent lesion in the left body of the mandible with focal radio-opacities located in the anterior aspect of the lesion. The lesion was causing root resorption, thinning of mandibular lower border and was extending from lower left 2 nd molar to lower left lateral incisor (Fig. 29.7d).

CT scan confirmed the presence of an expansile, lytic lesion with a characteristic 'ballooning distension of the buccal and lingual cortical plates'. It also showed scattered areas of radio-opacities \& multilocularity in the anterior region of the lesion and bi-cortical perforation along its posterior aspect.

'Ballooning distension' of the cortex with scattered areas of radio-opacities within a radiolucency was suggestive of secondary aneurysmal bone cyst arising in a pre-existing lesion (Fig. 29.7e).

Reconstructed CT scan showing bi-cortical perforation and lower border thinning at the mandibular body (Fig. 29.7f).

Aspiration: Figure 29.7c Dark venous blood of about 8-10 $\mathrm{ml}$ could readily be aspirated from the lesion.

Incisional Biopsy: Correlating the bloody venous aspirate, clinical \& radiological findings, a clinical diagnosis of the aneurysmal bone cyst was made. However, owing to the change in radiodensity in the anterior aspect of the lesion (as 
evident on CT scan), an incisional biopsy was performed to rule out the presence of a hybrid lesion or co-existing pathology.

Diagnosis: A histopathological diagnosis of secondary aneurysmal bone cyst arising in ossifying fibroma of the mandible has been made.

Surgical Plan: Considering the hybrid nature of the lesion, bi-cortical perforation, thinning of lower border, the proposed surgical plan was segmental mandibulectomy and reconstruction.

\section{Case Scenario 4 (Fig. 29.8)}

A 40-year-old female patient reported with a complaint of swelling over the lower jaw of 18 months duration and swelling over the right side of the upper jaw of 6 months duration.

\section{Clinical Examination}

A firm to hard swelling was noted in the mandibular anterior dentoalveolar region extending from right lower 2 nd premolar to lower left lateral incisor (Fig. 29.8a). There was a displacement of lower anterior teeth. Another similar firm swelling was noted in the right maxillary posterior dentoalveolar region in the region of missing right 1st maxillary molar (Fig. 29.8b). Overlying mucosa appeared normal in both the swellings.

\section{Pre-operative Diagnostic Work-up}

Imaging: Figure 29.8c Orthopantomogram revealed a unilocular radiolucency with a sclerotic border located in relation to the periapical region of right lower premolars. A poorly defined mixed lesion (radiolucent lesion with foci of radio opacities) was noted in the right maxillary posterior region.

Incisional Biopsy: An incisional biopsy of both the lesions was performed under local anaesthesia. Both the lesions were reported as central giant cell granulomas.

Biochemical Evaluation: Since synchronous giant cell granulomas are generally rare and as these lesions are histopathologically similar to browns tumour, biochemical evaluation was performed. Serum parathormone was remarkably elevated along with raised serum calcium levels, while renal function parameters were well within normal limits.

Ultrasound Evaluation of Neck: An ultrasound evaluation of neck revealed parathyroid adenoma on the left side.

Diagnosis: After correlating the biochemical and sonographic examination, a diagnosis of primary hyperparathyroidism is made. The osteolytic lesions of mandible and maxilla were diagnosed as Synchronous brown tumours of maxilla and mandible.

Surgical Plan: The patient was advised parathyroidectomy for primary hyperparathyroidism followed by the wait and watch policy for synchronous brown jaw tumours to undergo self-resolution. But spontaneous resolution the jaw lesions was very slow following eight months after parathyroidectomy and the patient wanted surgical removal of the brown jaw tumours. They were treated by marginal mandibulectomy and alveolectomy of posterior maxilla.

\section{References}

\section{Reactive Lesions of Oro-facial Soft Tissues}

1. Marx RE, Stern D. Oral and maxillofacial pathology: a rationale for diagnosis and treatment. Hanover Park, IL: Quintessence Publishing; 2012.

2. Eversole LR, Rovin S. Reactive lesions of the gingiva. J Oral Pathol Med. 1972;1(1):30-8.

3. Neville BW. Soft tissue tumours. In: Oral and maxillofacial pathology. London: Elsevier; 2009. p. 507-70.

4. Wick MR, McDermott MB, Swanson PE. Proliferative, reparative, and reactive benign bone lesions that may be confused diagnostically with true osseous neoplasms. Semin Diagn Pathol. 2014;31(1):66-88. WB Saunders

5. Hoch B, Montag A. Reactive bone lesions mimicking neoplasms. Semin Diagn Pathol. 2011;28(1):102-12. WB Saunders

6. Sternberg SS, Antonioli DA, Carter D, Mills SE, Oberman H. Diagnostic surgical pathology. 3rd ed. Philadelphia, PA: Lippincott Williams \& Wilkins; 1999.

7. Epivatianos A, Antoniades D, Zaraboukas T, Zairi E, Poulopoulos A, Kiziridou A, et al. Pyogenic granuloma of the oral cavity: comparative study of its clinicopathological and immunohistochemical features. Pathol Int. 2005;55:391-7.

8. Motamedi MH, Eshghyar N, Jafari SM, Lassemi E, Navi F, Abbas FM, Khalifeh S, Eshkevari PS. Peripheral and central giant cell granulomas of the jaws: a demographic study. Oral Surg Oral Med Oral Pathol Oral Radiol Endod. 2007;103:39-43.

9. Katsikeris N, Kakarantza-Angelopoulou E, Angelopoulos AP. Peripheral giant cell granuloma. Clinicopathologic study of 224 new cases and review of 956 reported cases. Int J Oral Maxillofac Surg. 1988;17(2):94-9.

10. Hernandez G, Lopez-Pintor RM, Torres J, de Vicente JC. Clinical outcomes of peri-implant peripheral giant cell granuloma: a report of three cases. J Periodontol. 2009;80:1184-91.

11. Hirshberg A, Kozlovsky A, Schwartz-Arad D, Mardinger O, Kaplan I. Peripheral giant cell granuloma associated with dental implants. J Periodontol. 2003;74(9):1380-4.

12. Subramanyam RV. Misnomers in oral pathology. Oral Dis. 2010;16(8):740-6.

13. Prasad S, Reddy S, Patil SR, Kalaburgi NB, Puranik RS. Peripheral ossifying fibroma and pyogenic granuloma. Are they interrelated? N Y State Dent J. 2008;74:50-2.

14. Buchner A, Hansen LS. The histomorphologic spectrum of peripheral ossifying fibroma. Oral Surg Oral Med Oral Pathol Oral Radiol. 1987;63(4):452-61. 
15. Mergoni G, Meleti M, Magnolo S, Giovannacci I, Corcione L, Vescovi P. Peripheral ossifying fibroma: a clinicopathologic study of 27 cases and review of the literature with emphasis on histomorphologic features. J Indian Soc Periodontol. 2015;19(1): 83-7.

16. Cuisia ES, Brannon RB. Peripheral ossifying fibroma-a clinical evaluation of 134 pediatric cases. Pediatr Dent. 2001;23(3): $245-8$

\section{Central Giant Cell Granuloma}

17. Yamaguchi T, Dorfman HD. Giant cell reparative granuloma: a comparative clinicopathologic study of lesions in gnathic and extragnathic sites. Int J Surg Pathol. 2001;9(3):189-200.

18. Schlorf RA, Koop SH. Maxillary giant cell reparative granuloma. Laryngoscope. 1977;87(1):10-7.

19. Chuong R, Kaban LB, Kozakewich H, Perez-Atayde A. Central giant cell lesions of the jaws: a clinicopathologic study. J Oral Maxillofac Surg. 1986;44(9):708-13.

20. Whitaker SB, Waldron CA. Central giant cell lesions of the jaws: a clinical, radiologic, and histopathologic study. Oral Surg Oral Med Oral Pathol Oral Radiol. 1993;75(2):199-208.

21. Eisenbud L, Stern M, Rothberg M, Sachs SA. Central giant cell granuloma of the jaws: experiences in the management of thirtyseven cases. J Oral Maxillofac Surg. 1988;46(5):376-84.

22. Tosco P, Tanteri G, Iaquinta C, Fasolis M, Roccia F, Berrone S, Garzino-Demo P. Surgical treatment and reconstruction for central giant cell granuloma of the jaws: a review of 18 cases. J CranioMaxillofac Surg. 2009;37(7):380-7.

23. Bataineh AB, Al-Khateeb T, Ma'amon AR. The surgical treatment of central giant cell granuloma of the mandible. J Oral Maxillofac Surg. 2002;60(7):756-61.

24. de Lange J, van den Akker HP, van den Berg H. Central giant cell granuloma of the jaw: a review of the literature with emphasis on therapy options. Oral Surg Oral Med Oral Pathol Oral Radiol Endod. 2007;104:603-15.

25. Pogrel AM. The diagnosis and management of giant cell lesions of the jaws. Ann Maxillofac Surg. 2012;2(2):102-6.

26. Suárez-Roa LM, Reveiz L, Ruíz-Godoy LR, Asbun-Bojalil J, Dávila-Serapio JE, Menjívar-Rubio AH, Meneses-García A. Interventions for central giant cell granuloma (CGCG) of the jaws. Cochrane Database Syst Rev. 2009;4:CD007404.

27. Vered M, Buchner A, Dayan D. Immunohistochemical expression of glucocorticoid and calcitonin receptors as a tool for selecting therapeutic approach in central giant cell granuloma of the jawbones. Int J Oral Maxillofac Surg. 2006;35(8):756-60.

28. Jacoway JH, Howell FV, Terry BC. Central giant cell granuloma: an alternative to surgical therapy. Oral Surg Oral Med Oral Pathol. 1988;66:572.

29. Terry BC, Jacoway JR. Management of central giant cell lesions: an alternative to surgical therapy. Oral Maxillofac Surg Clin North Am. 1994;6(3):579-600.

30. Abdo EN, Alves LC, Rodrigues AS, Mesquita RA, Gomez RS. Treatment of a central giant cell granuloma with intralesional corticosteroid. Br J Oral Maxillofac Surg. 2005;43(1): $74-6$.
31. Pogrel MA. Calcitonin therapy for central giant cell granuloma. J Oral Maxillofac Surg. 2003;61(6):649-53.

32. Kaban LB, Troulis MJ, Ebb D, August M, Hornicek FJ, Dodson TB. Antiangiogenic therapy with interferon alpha for giant cell lesions of the jaws. J Oral Maxillofac Surg. 2002;60(10):1103-11.

33. Da Silva NG, Carreira AS, Pedreira EN, Tuji FM, Ortega KL, Pinheiro JD. Treatment of central giant cell lesions using bisphosphonates with intralesional corticosteroid injections. Head Face Med. 2012;8(1):23.

34. Thomas D, Henshaw R, Skubitz K, Chawla S, Staddon A, Blay JY, Roudier M, Smith J, Ye Z, Sohn W, Dansey R. Denosumab in patients with giant-cell tumour of bone: an open-label, phase 2 study. Lancet Oncol. 2010;11(3):275-80.

35. Chawla S, Henshaw R, Seeger L, Choy E, Blay JY, Ferrari S, Kroep J, Grimer R, Reichardt P, Rutkowski P, Schuetze S. Safety and efficacy of denosumab for adults and skeletally mature adolescents with giant cell tumour of bone: interim analysis of an open-label, parallel-group, phase 2 study. Lancet Oncol. 2013;14(9):901-8.

36. Naidu A, Malmquist MP, Denham CA, Schow SR. Management of central giant cell granuloma with subcutaneous denosumab therapy. J Oral Maxillofac Surg. 2014;72(12):2469-84.

\section{Aneurysmal Bone Cyst}

37. Rosenberg AE, Nielson GP, Fletcher JA. Aneurysmal bone cyst. In: CDM F, Unni KK, Mertens F, et al., editors. WHO classification of tumours: pathology and genetics of head and neck tumours, vol. 9. 3rd ed. Lyon: IARC; 2005.

38. Struthers PJ, Shear M. Aneurysmal bone cyst of the jaws:(II). Pathogenesis. Int J Oral Maxillofac Surg. 1984;13(2):92-100.

39. Motamedi MH, Navi F, Eshkevari PS, Jafari SM, Shams MG, Taheri M, Abbas FM, Motahhari P. Variable presentations of aneurysmal bone cysts of the jaws: 51 cases treated during a 30-year period. J Oral Maxillofac Surg. 2008;66(10):2098-103.

40. Sun ZJ, Zhao YF, Yang RL, Zwahlen RA. Aneurysmal bone cysts of the jaws: analysis of 17 cases. J Oral Maxillofac Surg. 2010;68(9):2122-8.

\section{Brown Tumor}

41. Abdelrahman TE. Brown jaw tumors: challenges and outcomes. Int Surg J. 2017;4(11):3586-9.

42. Ullah E, Ahmad M, Ali SA, Redhu N. Primary hyperparathyroidism having multiple Brown tumors mimicking malignancy. Indian J Endocrinol Metab. 2012;16(6):1040.

43. Chami B, Benrachadi L, El Omri N, El Qatni M, El Wady W, El Mohtarim B. Brown tumor of the palate as first manifestation of primary hyperparathyroidism: a case report. Méd Buccale Chir Buccale. 2011;17(4):287-91.

44. Arunkumar KV, Kumar S, Deepa D. Brown tumor in mandible as a first sign of vitamin D deficiency: a rare case report and review. Indian J Endocrinol Metabo. 2012;16(2):310.

45. Scott SN, Graham SM, Sato Y, Robinson RA. Brown tumor of the palate in a patient with primary hyperparathyroidism. Ann Otol Rhinol Laryngol. 1999;108:91-4.

Open Access This chapter is licensed under the terms of the Creative Commons Attribution 4.0 International License (http://creativecommons. org/licenses/by/4.0/), which permits use, sharing, adaptation, distribution and reproduction in any medium or format, as long as you give appropriate credit to the original author(s) and the source, provide a link to the Creative Commons license and indicate if changes were made.

The images or other third party material in this chapter are included in the chapter's Creative Commons license, unless indicated otherwise in a credit line to the material. If material is not included in the chapter's Creative Commons license and your intended use is not permitted by statutory regulation or exceeds the permitted use, you will need to obtain permission directly from the copyright holder. 